\title{
Efecto de la herbivoría y coralivoría por peces en la supervivencia de corales trasplantados en el Caribe colombiano
}

\author{
Luis Chasqui-Velasco ${ }^{1}$, Elvira Alvarado Ch. ${ }^{2}$, Arturo Acero P. ${ }^{3}$ \& Fernando A. Zapata ${ }^{4}$ \\ 1 Parques Nacionales de Colombia, Territorial Suroccidente, Avenida 3G Norte 37-70, Cali, Colombia; luischasqui74@ \\ yahoo.com \\ 2 Universidad Jorge Tadeo Lozano, Bogotá, Colombia; elvira.alvarado@utadeo.edu.co \\ 3 Instituto de Ciencias Naturales, Universidad Nacional de Colombia, Bogotá, Colombia; aacero@invemar.org.co \\ 4 Departamento de Biología, Universidad del Valle, Cali, Colombia; fazapata@univalle.edu.co
}

\author{
Recibido 18-II-2006. Corregido 16-V-2006. Aceptado 15-I-2007.
}

\begin{abstract}
Effect of herbivorous and corallivorous fishes on the survival of transplanted corals in the Colombian Caribbean. To examine the effects of herbivorous and corallivorous fishes on the survival of transplanted colonies of Montastraea annularis, Diploria labyrinthiformis and Porites astreoides, both transplanted and native colonies were full-cage enclosed and compared to open (uncaged) colonies, while caging effects were assessed with a partial-cage (roof treatment). To evaluate if transplant stress increased the corals availability to fish predation, comparisons of fish foraging intensity among transplanted versus native colonies were made. To determine the density of herbivorous and corallivorous fishes on the transplants area visual censuses were made. The transient herbivorous fishes (Scaridae and Acanthuridae) were the most abundant fishes, and the corallivorous fishes (mainly Chaetodontidae) were the scarcest. A negative effect of territorial herbivorous fishes on $M$. annularis transplants survival was observed, mainly early on the study. Fish foraging intensity was similar on transplanted and native colonies, but differed among coral species, being lowest on D. labyrinthiformis. Fast macroalgal growth inside full-cages due to reduced fish grazing was observed. This caused partial bleaching and partial mortality in some colonies, mainly of $P$. astreoides. No significant difference in healthy tissue percentages among full-cage and uncaged colonies on $M$. annularis and D. labyrinthiformis was found, while in P. astreoides there were evident differences. The results indicate a damselfish negative effect on transplants survival early on the study, which can change depending on the fish and coral species involved. Results also indicate a fish grazing positive effect, caused by the reduction of coral-algae competition pressure, mainly on P. astreoides. Parrotfishes seem to affect corals survival both negatively through direct biting, and positively by controlling algal growth. Overall, coral transplant success was almost unaffected by fish foraging activity although several differences among coral species were obvious in relation to colony shape. Additionally, the interaction among herbivorous fish grazing and coral-algae competition balance appear important in determining transplant survival. Rev. Biol. Trop. 55 (3-4): 825-837. Epub 2007 December, 28.
\end{abstract}

Key words: herbivorous fishes, corallivorous fishes, Montastraea annularis, Diploria labyrinthiformis, Porites astreoides, coral-fish interaction.

Muchos arrecifes costeros en todo el mundo enfrentan actualmente una progresiva fase de cambio (McCook 1999). Este cambio se caracteriza por la reducción en la cobertura coralina, la proliferación de algas bentónicas y la disminución de la biodiversidad local (Lapointe 1989, Hughes 1994). Entre las causas (Brown 1997a, Wilkinson 2000) están factores antropogénicos como el aumento del aporte de nutrientes y contaminantes al mar y la sobreexplotación pesquera en las costas, igualmente, factores naturales como las perturbaciones biológicas de gran escala eg. mortalidad masiva de Diadema antillarum (Philippi, 1845) y el blanqueamiento coralino por calentamiento del agua (Carpenter 1990, Brown 1997b). 
Desde los ochentas algunos investigadores advirtieron el deterioro progresivo de los arrecifes alrededor del Archipiélago del Rosario en el Caribe Colombiano (Alvarado et al. 1986, Ramírez 1986). En menos de veinte años se pasó de comunidades arrecifales bien desarrolladas, con grandes extensiones de coral del género Acropora en buen estado y 48 especies de escleractíneos, a un paisaje formado por cementerios de Acropora palmata (Lamarck, 1816), lechos de cascajo de A. cervicornis (Lamarck, 1816) cubiertos por algas y menos de 30 especies de escleractíneos (Sarmiento et al. 1990, Schonwald 1998, obs. pers. 1997). Paralelamente, aumentó la abundancia de algas pardas, principalmente de los géneros Dictyota y Lobophora (Alvarado et al. 1986). El enriquecimiento nutritivo de las aguas, la pesca con dinamita, el aumento del turismo y la extracción de organismos y material para construcción, son algunas causas de este deterioro (Alvarado et al. 1986, Alvarado y Corchuelo 1992).

En respuesta al declive de los arrecifes coralinos, ha surgido interés en el desarrollo de estrategias para su recuperación. Una herramienta útil es el trasplante de corales de áreas poco perturbadas a áreas perturbadas (Guzmán 1991), considerado un buen método para el mejoramiento y la preservación de la biodiversidad en arrecifes coralinos. El trasplante permite saltar sobre los estadíos iniciales de la sucesión natural, iniciando y acelerando la recolonización y creación del andamio estructural en arrecifes deteriorados (Bouchon et al. 1981, van Treeck y Schuhmacher 1997).

El conocimiento de los factores que determinan la supervivencia de corales trasplantados está aún en desarrollo; sin embargo, se cree que la herbivoría y la depredación por peces pueden influir de manera importante (García 1994, Clark y Edwards 1995, van Treeck y Schuhmacher 1997). La herbivoría afecta la diversidad, distribución, abundancia y productividad de las comunidades de algas, determinando la intensidad de la competencia con los corales trasplantados, lo cual puede influir indirectamente en el éxito del trasplante (Ogden y Lobel 1978, Glynn 1990). Los estudios en el
Caribe sobre el efecto de los peces herbívoros en la estructura de la comunidad bentónica sugieren que la reducción en la presión de forrajeo afecta negativamente la supervivencia de los corales (Hay y Taylor 1985, Carpenter 1986, Lewis 1986). La depredación por peces, en cambio, puede afectar directamente los corales limitando el crecimiento, abundancia y distribución de algunas especies (Neudecker 1979, Cox 1986, Guzmán 1988, Littler et al. 1989). La pérdida de tejido coralino por la mordedura constante de algunos peces, puede reducir las posibilidades de supervivencia de las colonias trasplantadas, disminuyendo el éxito del trasplante (Miller y Hay 1998).

A pesar de la importancia de los peces como depredadores de coral y de su influencia en la interacción competitiva coral-alga (Glynn 1990), no hay claridad sobre su efecto en la supervivencia de corales trasplantados. El objetivo de esta investigación fue determinar si los peces herbívoros y coralívoros afectan la supervivencia de colonias trasplantadas de Montastraea annularis (Ellis y Solander, 1786), Diploria labyrinthiformis (Linnaeus, 1758) y Porites astreoides (Lamarck, 1816). Específicamente se querían resolver las siguientes preguntas: ¿Cómo afectan los peces herbívoros la supervivencia de corales trasplantados? ¿Cómo influyen los peces coralívoros en dicha supervivencia? ¿Es más importante el efecto de los peces herbívoros o el de los coralívoros? ¿Altera el trasplante la interacción normal de estos peces con los corales? Si existe algún efecto ¿Es igual para las tres especies?

\section{MATERIALES Y MÉTODOS}

Área de estudio: el estudio se realizó en la barrera de Isla Grande, Archipiélago del Rosario, Caribe colombiano (10¹1'04" N, $\left.75^{\circ} 43^{\prime} 51^{\prime}, \mathrm{W}\right)$. Los corales trasplantados se ubicaron en el frente arrecifal, una terraza de amplitud variable $(50-200 \mathrm{~m})$ entre $\operatorname{los} 8$ y $14 \mathrm{~m}$ de profundidad. Esta zona consiste de fragmentos semiconsolidados de A. cervicornis y grandes colonias de Montastraea sp., 
asociadas a Colpophyllia natans (Houttuyn, 1772), Agaricia sp., Mycetophyllia sp., D. labyrinthiformis y algunas colonias de $P$. astreoides. El frente arrecifal presenta características físicas (profundidad, pendiente y tipo de sustrato) y biológicas (composición y densidad de corales, peces territoriales, algas y bioerodadores) similares a las del arrecife donante (Alvarado et al., en prep.).

Abundancia de peces herbívoros y coralívoros: en febrero de 1997 se hizo un reconocimiento de los peces herbívoros y coralívoros en el frente arrecifal (Cuadro 1). Para determinar su abundancia se realizaron censos visuales por el método de transecto cuadrante simultáneo (Fowler 1987). Utilizando un transecto de $50 \mathrm{~m}$ se registraron los individuos observados en frente del buzo hasta un metro a cada lado. Los censos se realizaron entre las 8:30 am y las $3: 30 \mathrm{pm}$ en junio $(\mathrm{n}=7)$, agosto $(\mathrm{n}=7)$, octubre $(n=12)$ y diciembre $(n=4)$ de 1997 y en abril $(n=3)$ de 1998.

Intensidad de forrajeo: para estimar la intensidad de forrajeo de los peces sobre las especies de coral trasplantadas y saber si cambió con relación al trasplante, se hicieron observaciones en colonias trasplantadas y nativas (es decir, propias del arrecife receptor) de cada especie durante cinco minutos. Se realizaron entre 45 y 47 períodos de observación para cada especie y tratamiento a lo largo del estudio, donde se registró el número de mordidas y las especies de peces observadas mordiendo el coral. Con estas observaciones se obtuvo un estimativo de la intensidad del mordisqueo de los peces en los corales expresado como el promedio de mordeduras ocurridas durante cinco minutos para colonias trasplantadas y nativas por especie.

Para evaluar si existió un aumento en la presión de forrajeo por peces asociado con el estrés generado durante el trasplante y si fue diferente entre las especies de coral, se compararon los promedios de mordedura entre colonias trasplantadas y nativas de las tres especies mediante un análisis de varianza de dos vías
CUADRO 1

Peces herbivoros y coralivoros observados en la barrera de Isla Grande

TABLE 1

Herbivorous and corallivorous fishes observed in the Isla Grande barrier

Herbívoros móviles

Acanthuridae

Acanthurus bahianus Castelnau

A. chirurgus (Bloch)

A. coeruleus Schneider

Scaridae

Scarus croicensis Bloch

S. taeniopterus Desmarest

S. vetula Schneider

Sparisoma atomarium (Poey)

S. aurofrenatum (Valenciennes)

S. chrysopterum (Bloch y Schneider)

S. rubripinne (Valenciennes)

S. viride (Bonnaterre)

Herbívoros territoriales

Pomacentridae

Microspathodon chrysurus (Cuvier)

Stegastes diencaeus (Jorden y Rutter)

S. dorsopunicans (Poey)

S. leucostictus (Müller y Troschel)

S. partitus (Poey)

S. planifrons (Cuvier)

S. variabilis (Castelnau)

Coralívoros

Chaetodontidae

Chaetodon capistratus Linnaeus

C. ocellatus Bloch

C. striatus Linnaeus

Tetraodontidae

Canthigaster rostrata (Bloch) 
en los datos transformados por raíz cuadrada (Zar 1996). Se realizaron pruebas post-anova de Newman-Keuls para identificar las especies diferentes.

Reducción de herbivoría y depredación: el experimento para evaluar el efecto de reducir la herbivoría y la depredación sobre los corales trasplantados consistió de tres tratamientos: una jaula de malla plástica (luz de malla de 1 $\mathrm{cm}^{2}$ ) para excluir los peces de un área de 0.16 $\mathrm{m}^{2}$ cubriendo los corales; un techo de la misma malla para controlar el efecto de sombra debido a la jaula; por último, se monitorearon colonias sin ningún tratamiento para controlar los efectos de la manipulación. Para descartar un posible efecto del estrés del trasplante sobre los resultados del experimento de exclusión, se instalaron los mismos tratamientos en colonias no perturbadas nativas. El tamaño de muestra fue de seis para cada tratamiento por especie, tanto en colonias trasplantadas como en nativas. Los tratamientos se instalaron en abril de 1997 y se monitorearon cada dos meses hasta abril de 1998. Para el monitoreo se utilizó una videocámara con la que se filmaron desde arriba las colonias de las tres especies. Posteriormente, cada colonia fue dibujada desde la pantalla en un acetato destacando las partes saludables, blanqueadas y muertas. En $P$. astreoides se indicaron además las partes con cubiertas de mucus. Los acetatos fueron colocados sobre una nube de puntos uniformemente distribuidos, y se registró el número de puntos ubicados en cada parte del dibujo y el número de puntos totales. Esto permitió obtener porcentajes de la cantidad de tejido sano, blanqueado, con cubiertas de mucus y muerto para cada colonia en cada fecha de muestreo.

El porcentaje de tejido coralino sano se obtuvo restando del total los porcentajes de tejido muerto, blanqueado y con cubiertas de mucus, según el caso. Para evaluar el efecto de la reducción de la presión de forrajeo de los peces herbívoros y coralívoros sobre los trasplantes, se compararon los porcentajes de tejido coralino sano de las colonias en los tratamientos de jaula y de control a lo largo de un año. Las comparaciones se hicieron mediante pruebas de Mann-Whitney para cada fecha de muestreo. Para analizar la posible influencia del estrés del trasplante en los resultados del experimento de reducción de herbivoría y coralivoría, se compararon las colonias trasplantadas de control con las colonias nativas de control. Las comparaciones para cada especie en cada fecha de muestreo se hicieron con pruebas de MannWhitney. Finalmente, para evaluar si existió algún efecto del montaje experimental causado por las jaulas se comparó entre colonias en el tratamiento de techo y colonias control, tanto en colonias trasplantadas como en nativas. Las comparaciones se hicieron por medio de pruebas de Mann-Whitney para cada especie y fecha de muestreo.

\section{RESULTADOS}

Abundancia e intensidad de forrajeo por peces: los peces herbívoros móviles (Scaridae y Acanthuridae) fueron el grupo más abundante de los tres estudiados alcanzando el $55.6 \%$ de un total de 3017 peces observados y el 57.6 $\%$ del total de peces herbívoros. Se destacan las especies Scarus croicensis (Bloch, 1790) y Scarus taeniopterus (Desmarest, 1831) como las más abundantes en este grupo (21.5 ind/100 $\mathrm{m}^{2}$ ). Los herbívoros territoriales (Stegastes, Pomacentridae) representaron el $40.8 \%$ del total de peces y el $42.4 \%$ del total de peces herbívoros, con Stegastes planifrons (Cuvier, 1830) como el más abundante del grupo con $15.6 \mathrm{ind} / 100 \mathrm{~m}^{2}$. Los peces coralívoros representaron solamente el $3.6 \%$ del total; Canthigaster rostrata (Bloch, 1786) fue la especie más abundante dentro del grupo con $1.4 \mathrm{ind} / 100 \mathrm{~m}^{2}$ (Fig. 1).

La actividad alimentaria sobre los corales estudiados fue baja tanto en colonias trasplantadas como en nativas; solamente en el $18 \%$ de los muestreos para las tres especies y los dos tratamientos $(n=272)$ se observó mordisqueo de los peces en los corales. Se registraron diferencias en la intensidad de forrajeo por peces entre las especies de coral, y en dos de las 


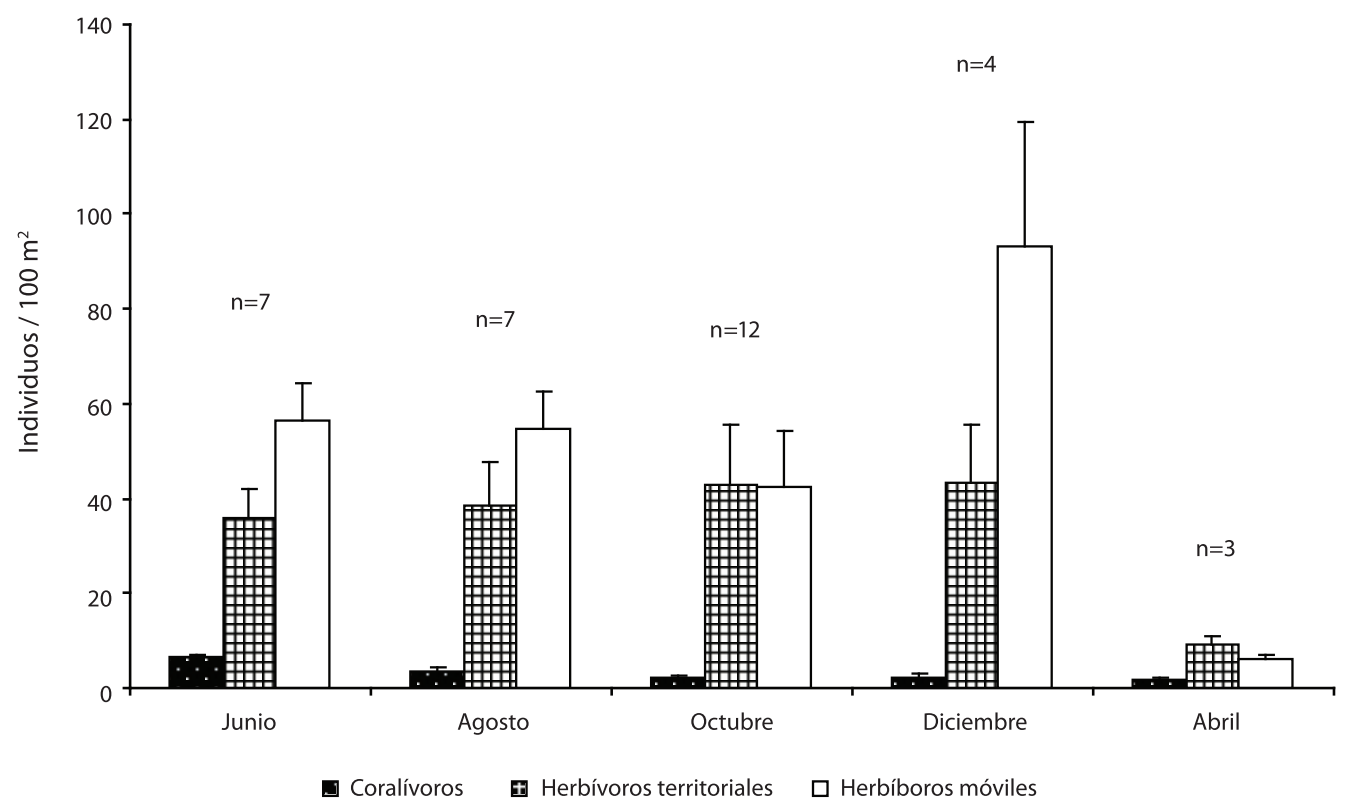

Fig. 1. Abundancia $(\mathrm{X} \pm \mathrm{EE})$ de los peces herbívoros y coralívoros en la zona del frente arrecifal de la barrera de Isla Grande.

Fig. 1. Herbivorous and corallivorous fish abundance $(\mathrm{X} \pm \mathrm{SEM})$ in the Isla Grande fore-reef terrace.

tres especies estudiadas hubo diferencias entre colonias trasplantadas y nativas (Fig. 2). La intensidad de forrajeo por peces en $M$. annularis y $P$. astreoides fue significativamente mayor que en D. labyrinthiformis (Newman-Keuls, $\mathrm{p}<0.05)$. Mientras que en $D$. labyrinthiformis los peces forrajearon con igual intensidad en colonias trasplantadas y nativas, en $M$. annularis forrajearon más en colonias trasplantadas y en $P$. astreoides forrajearon más en colonias nativas (Fig. 2).

Los peces observados con mayor frecuencia forrajeando sobre los corales estudiados fueron los herbívoros territoriales Stegastes diencaeus (Jordan \& Rutter, 1897) y S. planifrons, seguidos por juveniles de $S$. croicensis y $S$. taeniopterus, que corresponden al grupo de los herbívoros móviles. De los peces coralívoros, solamente se observó depredación por Chaetodon capistratus (Linnaeus, 1758) (Fig. 3).

Reducción de herbivoría y depredación: las jaulas excluyeron los peces herbívoros móviles y los peces coralívoros (C. rostrata, Chaetodontidae), pero no a los peces herbívoros territoriales. La reducción en la intensidad de forrajeo debida a la exclusión de acanthúridos y scáridos alteró notablemente la comunidad algal dentro de las jaulas. Se observó un aumento en la cobertura de macroalgas, principalmente de los géneros Dictyota, Halimeda y Peyssonellia, y las especies Lobophora variegata (Lamouroux) Womersley ex Oliveira, Anadyomene stellata (Wulfen) C. Agardh, Amphiroa tribulus (Ellis \& Solander) Lamouroux, Amphiroa fragilissima (Linnaeus) Lamouroux, Kallymenia westi (Agardh) y Wrangelia argus (Montagne) Montagne. No obstante, el efecto del aumento de las macroalgas sólo fue evidente en $M$. annularis, donde el sobrecrecimiento de $A$. fragillisima causó mortalidad del $5 \%$ en una colonia al impedir la entrada de luz; y en $D$. labyrinthiformis donde A. stellata causó blanqueamiento en algunas colonias (10\% del tejido vivo) al final del estudio por la misma razón. En las colonias de techo y control, también se redujo 


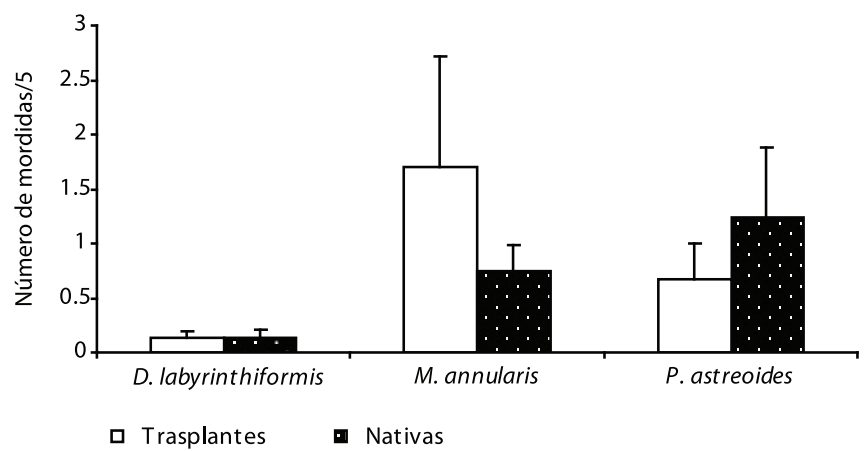

Fig. 2. Intensidad de forrajeo $(\mathrm{X} \pm \mathrm{EE})$ por peces en los corales Diploria labyrinthiformis, Montastraea annularis y Porites astreoides, en colonias trasplantadas y nativas.

Fig. 2. Fish foraging intensity $(\mathrm{X} \pm \mathrm{SEM})$ on transplanted and native colonies of Diploria labyrinthiformis, Montastraea annularis and Porites astreoides coral species.

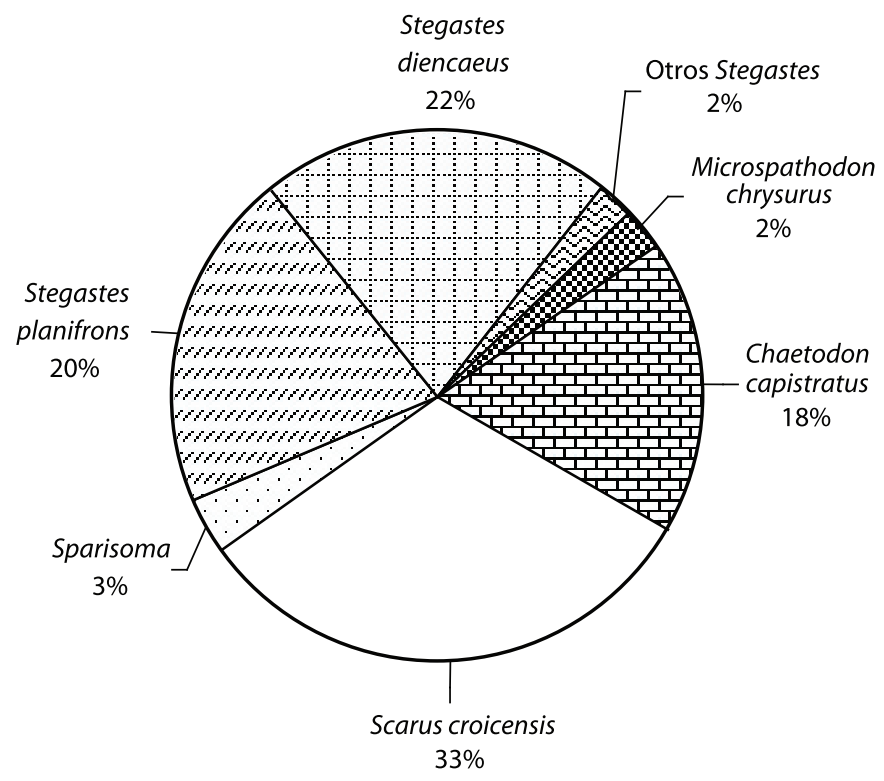

Fig. 3. Especies de peces observadas mordisqueando las colonias trasplantadas y nativas de Montastraea annularis, Diploria labyrinthiformis y Porites astreoides. El número bajo el nombre de la especie indica su contribución relativa (\%) al total de mordidas $(\mathrm{N}=210)$ durante 1360 minutos de observación.

Fig. 3. Fish species observed biting on transplanted and native colonies of Montastraea annularis, Diploria labyrinthiformis and Porites astreoides. Numbers below species name indicates its relative contribution (\%) at the total bites $(\mathrm{N}=210)$ on 1360 minutes of observation. la intensidad de forrajeo pero de manera natural, debido a la defensa activa de los pomacéntridos territoriales.

\section{Montastraea annularis:} en esta especie el estrés del trasplante pareció afectar la supervivencia de las colonias. En las colonias trasplantadas de control se observaron porcentajes de tejido coralino sano menores, en comparación con colonias nativas también de control al inicio del estudio (mayo, Mann-Whitney en los rangos del porcentaje de coral sano, $\mathrm{p}<0.05$ ). A través del estudio el porcentaje de tejido coralino sano de las colonias trasplantadas de control aumentó ligeramente, disminuyendo la diferencia con las colonias nativas, lo que indicó una recuperación de las colonias trasplantadas al estrés inicial causado por el trasplante (Fig. 4).

El efecto de los peces en las colonias trasplantadas no fue muy claro en esta especie; sin embargo, en colonias nativas los peces parecen jugar un papel importante. En las colonias trasplantadas dentro de las jaulas, se observaron porcentajes de tejido coralino sano mayores que en las colonias de control a través del estudio (Mann-Whitney $\mathrm{p}<0.05$ ), sin una tendencia clara hacia el aumento o reducción del porcentaje de tejido coralino sano en ninguno de los dos tratamientos (Fig. 4). El caso contrario se observó en 

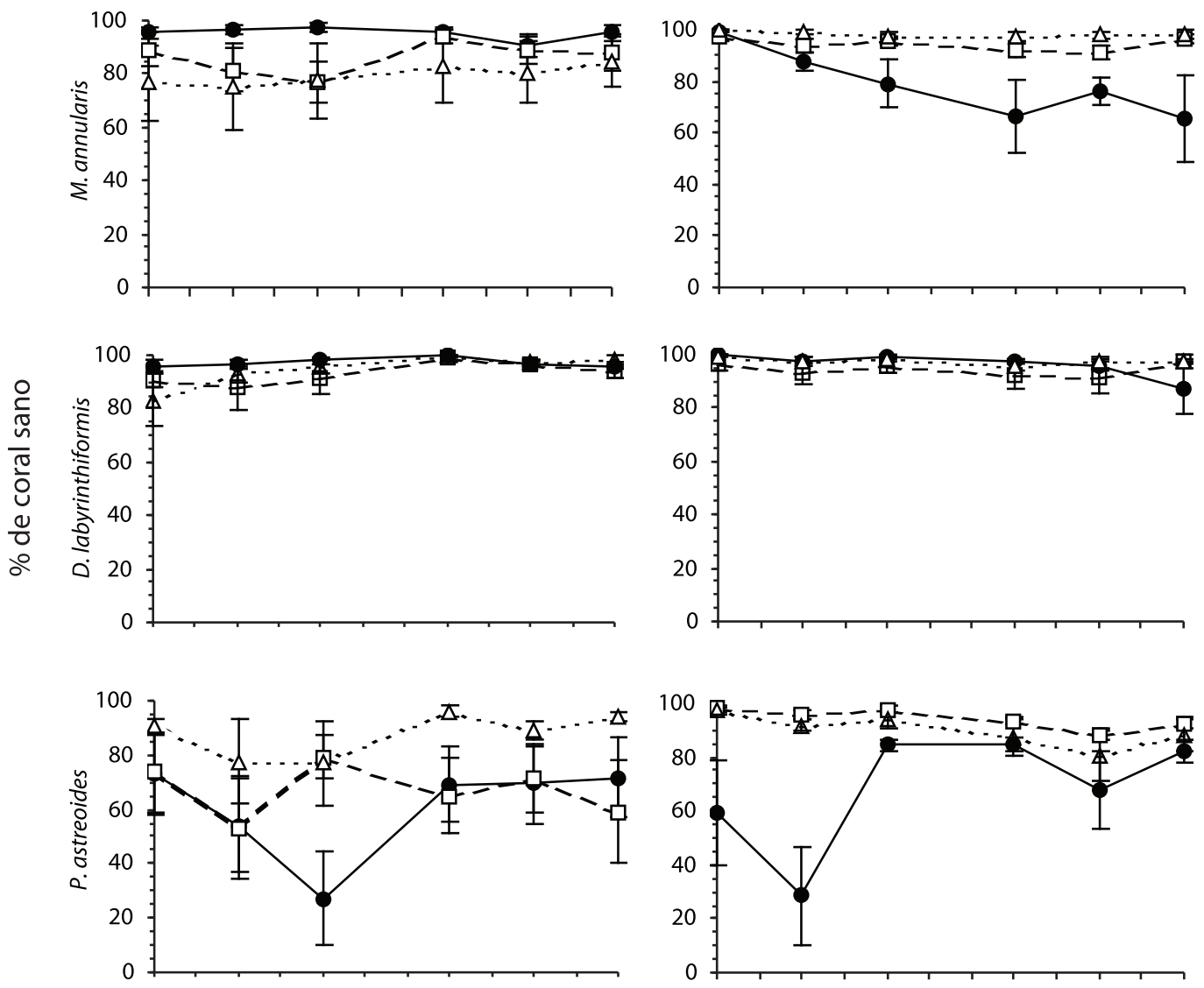

$$
\longrightarrow \text { Jaula } \quad--\square-- \text { Techo } \quad \cdots \Delta-\text { - Control }
$$

Fig. 4. Variación temporal del porcentaje de tejido coralino sano $(\mathrm{X} \pm \mathrm{EE})$ de colonias trasplantadas y nativas de Montastraea annularis, Diploria labyrinthiformis y Porites asteroides bajo condiciones naturales (Control), exclusión de peces (Jaula) y control de jaula (Techo).

Fig. 4. Temporary variation of the percentage of healthy coralline tissue $(\mathrm{X} \pm \mathrm{SEM})$ of transplanted and native colonies of Montastraea annularis, Diploria labyrinthiformis and Porites astreoides under unmanipulated (control), full-cage (fish exclusion) and roof-only cage (full-cage control) conditions along the study.

las colonias nativas, donde se presentaron porcentajes de tejido coralino sano mayores en las colonias de control y menores en el tratamiento de jaulas a partir de julio (MannWhitney $\mathrm{p}<0.05$ ). Además, en estas últimas se observó una marcada tendencia a la reducción en el porcentaje de tejido coralino sano (Fig. 4). Al final del estudio se registró la muerte total de una colonia donde aparecía establecido un jardín de algas filamentosas de S. planifrons, y aunque no se hizo un seguimiento cuantitativo del proceso si pudo observarse el mordisqueo activo y frecuente del pez damisela sobre el tejido coralino vivo, lo que sugiere una relación de causa-efecto entre la muerte del coral y el establecimiento del jardín de algas.

Las comparaciones en el porcentaje de tejido coralino sano entre techos y controles, 
no mostraron ningún efecto del montaje experimental tanto en las colonias trasplantadas como en las nativas (Mann-Whitney $\mathrm{p}>0.05$ ). Durante el estudio se registraron 40 marcas de mordidas de peces loro en los trasplantes expuestos y una marca en colonias nativas.

Diploria labyrinthiformis: en esta especie también se observó un efecto del estrés causado por el trasplante, pero la recuperación fue más rápida que en $M$. annularis (Fig. 4). Las colonias trasplantadas de control presentaron porcentajes de tejido coralino sano menores en comparación con colonias nativas de control al inicio del estudio (mayo, Mann-Whitney, $\mathrm{p}<0.05$ ). El porcentaje de tejido coralino sano de las colonias trasplantadas de control aumentó rápidamente, alcanzando en septiembre valores similares a las colonias nativas de control, indicando la recuperación de las colonias trasplantadas al estrés inicial causado por el trasplante (Fig. 4).

No hubo un efecto evidente de los peces en D. labyrinthiformis, tanto en colonias trasplantadas como en nativas. Al comparar las colonias trasplantadas en el tratamiento de jaulas con las colonias trasplantadas de control solo se encontraron diferencias en mayo (MannWhitney, $\mathrm{p}<0.05$; Fig. 4), que pueden deberse a efectos del trasplante. En las colonias nativas no se encontraron diferencias a lo largo del período de estudio (Mann-Whitney, $\mathrm{p}>0.05$ ).

Al comparar el porcentaje de tejido coralino sano entre el tratamiento de techos y los controles, no se encontraron diferencias en todo el período de estudio tanto en colonias trasplantadas como en nativas, indicando que no hubo ningún efecto del montaje experimental (Mann-Whitney, $\mathrm{p}>0.05$ ). Durante el estudio se registraron cinco cicatrices de forrajeo de peces loro en las colonias trasplantadas expuestas y una en colonias nativas, sugiriendo una mínima depredación por peces en esta especie.

Porites astreoides: en esta especie las colonias trasplantadas de control presentaron porcentajes de tejido coralino sano menores que las colonias nativas de control en mayo, julio y septiembre (Mann- Whitney, $\mathrm{p}<0.05$ ), indicando un posible efecto del estrés del trasplante. Sin embargo, la variación en el porcentaje de tejido coralino sano observada en las colonias nativas de control, sugiere una mayor variación natural en la salud de esta especie comparada con $M$. annularis y D. labyrinthiformis (Fig. 4).

Las colonias trasplantadas en el tratamiento de jaulas tuvieron porcentajes de tejido coralino sano menores que las colonias trasplantadas de control durante todo el período de estudio (Mann-Whitney, $\mathrm{p}<0.05$ ); sin embargo, el comportamiento de la variable no indica una tendencia clara que pueda relacionarse con el efecto del tratamiento (Fig. 4). En las colonias nativas el porcentaje de tejido coralino sano del tratamiento de jaulas fue menor entre mayo y julio en comparación con las colonias de control (Mann-Whitney, $\mathrm{p}<0.05$ ), aumentando significativamente en septiembre.

El efecto del montaje experimental no fue evidente en las colonias nativas, pero sí en los trasplantes. Al comparar las colonias nativas de techo y control no se encontraron diferencias durante todo el estudio (MannWhitney, $\mathrm{p}>0.05$ ); sin embargo, en las colonias trasplantadas el tratamiento de techos presentó porcentajes de tejido coralino sano menores que los controles durante casi todo el estudio (Mann-Whitney, $\mathrm{p}<0.05$; Fig. 4). Durante el estudio se registraron 50 marcas de forrajeo de peces loro en colonias trasplantadas y nueve en colonias nativas, principalmente en el borde de colonias de techo.

\section{DISCUSIÓN}

Abundancia e intensidad de forrajeo: los resultados de este estudio sugieren que el forrajeo de los peces loro y el mordisqueo de los peces damisela, afectan en mayor medida la supervivencia de colonias coralinas que el forrajeo de los peces coralívoros. La mayoría de las mordeduras en los corales fueron hechas por juveniles de $S$. croicensis y $S$. taeniopterus, indicando un efecto de depredación 
importante de estas especies sobre los corales. La importancia del forrajeo de ciertos scáridos sobre algunos corales ha sido mencionada por Miller y Hay (1998), quienes obtuvieron una mortalidad del $56 \%$ en $48 \mathrm{~h}$, debido al forrajeo de Sparisoma viride (Bonnaterre, 1788) y $S$. aurofrenatum (Valenciennes, 1840) sobre colonias trasplantadas sin protección.

El forrajeo más intenso y continuo fue hecho por peces herbívoros territoriales durante una semana después del trasplante; estos peces establecieron sus territorios sobre las colonias trasplantadas mordiendo el tejido coralino. Las observaciones durante los primeros días del trasplante sugieren que estos peces influyeron de manera importante en la supervivencia inicial de las colonias de dos maneras: (1) expulsando agresivamente a otros peces que rondaban los trasplantes, entre ellos algunos depredadores potenciales como los scáridos y chaetodóntidos, reduciendo así el forrajeo y (2) mordisqueando continuamente algunas colonias de $M$. annularis, causando mortalidad parcial en las colonias afectadas.

Knowlton et al. (1990) encontraron que el efecto negativo de $S$. planifrons en la supervivencia de corales bajo estrés, puede ser tan importante o más que el de algunos coralívoros especializados. Potts (1977), al trasplantar fragmentos del coral Acropora palifera (Lamarck, 1816), reportó alta mortalidad debido al establecimiento de territorios de Dischistodus perspicillatus (Cuvier, 1830). Este autor no observó forrajeo por peces en los corales dentro de los territorios, pero sí en aquellos que no fueron colonizados por peces damisela. Kaufman (1977) documentó las actividades de S. planifrons sobre $M$. annularis y A. cervicornis, como una manera de obtener sustrato libre adicional para el crecimiento de las algas filamentosas, y su importancia como un factor negativo en la salud de los corales.

Los resultados muestran una presión de forrajeo diferente en los corales estudiados, siendo mayor en $M$. annularis y $P$. astreoides que en $D$. labyrinthiformis; sin embargo, las causas aún son desconocidas. En estudios previos, realizados en varias localidades y con especies y procedimientos distintos, también se han registrado diferencias en el forrajeo entre especies de coral sin que haya una explicación satisfactoria (Neudecker 1979, Miller y Hay 1998); no obstante, parece que las diferencias morfológicas son importantes.

Wellington (1982) menciona la importancia de la forma de la colonia en el escape al forrajeo por peces, donde las especies ramificadas son preferidas a las masivas. En nuestro estudio las tres especies trasplantadas son masivas, sin embargo una mayor curvatura superficial de $M$. annularis por su crecimiento columnar en oposición a una topografía más plana de $P$. astreoides y $D$. labyrinthiformis, podría facilitar un mayor forrajeo de los peces raspadores como $S$. croicensis y $S$. taeniopterus en la primera.

En el caso de los pomacéntridos territoriales parece haber una cierta especificidad en su relación con los corales. Wellington (1982) encontró que Stegastes acapulcoensis (Fowler, 1944) prefirió establecer sus tapetes de algas sobre Pavona gigantea (Verrill, 1869) en vez de Pocillopora damicornis (Linnaeus, 1758); él sugirió que la mayor disponibilidad de superficies verticales amplias hacen de la primera un mejor sustrato para la postura. En nuestro estudio, el crecimiento columnar de $M$. annularis crea superficies verticales que podrían ser aprovechadas como sitios de postura por los peces damisela; en contraste, la topografía más plana de $D$. labyrinthiformis y la forma de plato de $P$. astreoides las hacen poco adecuadas. De hecho, las colonias mordisqueadas de $M$. annularis presentaban grandes parches de esqueleto desnudo con orientación vertical. Esto contribuye a explicar el mayor efecto del mordisqueo de los pomacéntridos territoriales sobre $M$. annularis.

Reducción de herbivoría y depredación: los resultados del experimento de exclusión de peces sugieren que no existió un efecto importante de los peces herbívoros móviles ni de los peces coralívoros en la supervivencia al trasplante de $M$. annularis y D. labyrinthiformis, pero sí en $P$. astreoides (Fig 4). El escape a la 
depredación de los peces que se esperaba con la instalación de las jaulas no se logró en su totalidad, ya que los peces damisela pudieron entrar y mordisquear por igual las colonias protegidas y las expuestas. Igualmente, la protección de los pomacéntridos territoriales a las colonias expuestas pudo reducir las diferencias en el forrajeo con respecto a las colonias protegidas, enmascarando el efecto de los tratamientos.

Dentro de las jaulas hubo un incremento notorio en la abundancia de las macroalgas, y en consecuencia una presión competitiva mayor sobre los corales (debida al sobrecrecimiento y sombreado). En el caso de las colonias trasplantadas de $M$. annularis, debido a su crecimiento columnar el tejido vivo quedaba a una altura considerable del sustrato, permitiendo que escapara a una interacción fuerte con las macroalgas. D. labyrinthiformis en cambio sufrió algunos blanqueamientos debido al aumento de macroalgas, lo que fue evidente dado que al retirar los tejidos de las macroalgas podía observarse debajo un área de tejido coralino blanqueado de similar forma y tamaño. Sin embargo, la cantidad de tejido blanqueado no fue suficiente para crear diferencias significativas en el porcentaje de tejido coralino sano entre colonias protegidas y expuestas.

En $P$. astreoides los porcentajes de tejido coralino sano menores en colonias protegidas que en colonias expuestas al forrajeo de los peces, indican que hubo algún efecto de la exclusión de los peces en la supervivencia de los trasplantes. Estos resultados se debieron principalmente al blanqueamiento, sugiriendo que $P$. astreoides fue afectada principalmente por el aumento de la competencia con macroalgas dentro de las jaulas. La forma de plato de las colonias de $P$. astreoides permitió que el tejido vivo se encontrara más cerca del sustrato, facilitando el sobrecrecimiento por las macroalgas con el consecuente efecto de sombreado y acentuando la presión de competencia con los organismos bentónicos en general. Lo anterior sugiere que la morfología de las colonias trasplantadas puede jugar un papel importante en su supervivencia, donde las especies con crecimiento columnar podrían tener mayor éxito que las especies aplanadas, dado que el tejido coralino estaría más alejado del sustrato.

Los resultados muestran un escaso efecto de la depredación por peces coralívoros sobre la supervivencia de las colonias trasplantadas, sin embargo el mordisqueo de los peces loros y damiselas sí tuvo una importante influencia que cambió entre las especies trasplantadas. En $P$. astreoides, las marcas de forrajeo de peces loro y las mordeduras observadas en algunas colonias expuestas, sugieren que la depredación contribuyó para la diferencia en el porcentaje de coral sano entre colonias trasplantadas protegidas y expuestas al forrajeo. En D. labyrinthiformis, las escasas mordeduras tanto en colonias trasplantadas como en nativas expuestas, no mostraron diferencias al compararlas con colonias protegidas. Por otra parte, la ausencia de mordisqueo de los peces damisela sobre $D$. labyrinthiformis indica que su efecto sobre esta especie, si lo hay, se remitiría a prevenir el forrajeo de los depredadores potenciales. En M. annularis fue más marcado el efecto de la depredación por peces, debida a las mordeduras de los peces loro y especialmente al mordisqueo de los pomacéntridos territoriales. Este último factor causó la mortalidad total de una colonia nativa de jaula, indicando su importancia para la supervivencia de colonias individuales.

La mayoría de estudios en el Caribe sobre el rol ecológico de los acanthúridos y scáridos, mencionan su importancia para el mantenimiento de las comunidades bentónicas arrecifales dominadas por corales escleractíneos (Brock 1979, Lewis 1986, Hughes 1994). Sin embargo, algunas especies de peces loro pueden consumir coral vivo (Frydl 1979, Bruggemann et al. 1994, Bruckner y Bruckner 1998) y esa depredación puede tener efectos importantes sobre los corales al nivel de comunidad (Miller y Hay 1998). En nuestro estudio se observaron los dos efectos antagónicos del forrajeo de los peces loro: el forrajeo directo sobre corales trasplantados y nativos con un efecto negativo para la supervivencia de colonias individuales, y el efecto positivo indirecto del forrajeo sobre las algas para la salud de los corales. 
El efecto del forrajeo directo de peces loro sobre los corales es un tema de debate. Por ejemplo, Bruggemann et al. (1994) afirman que las mordeduras funcionan más como marcadores territoriales que con fines alimenticios, en cambio Bruckner y Bruckner (1998) registran la mortalidad de colonias completas por el mordisqueo continuo de peces loro. Miller y Hay (1998) sugieren que el efecto positivo indirecto de los peces loro sobre los corales al reducir la competencia de las macroalgas, puede ser opacado por su efecto negativo al depredar directamente sobre los corales.

En conclusión, la influencia de los peces en la supervivencia de los trasplantes parece depender en gran medida de las especies de coral y de peces involucrados, al menos en la etapa inicial. Los peces herbívoros territoriales pueden tener un efecto más significativo para la salud de los trasplantes que los peces coralívoros, especialmente en los primeros días del trasplante. La influencia de los peces herbívoros móviles (principalmente Scaridae) puede presentarse de dos formas: (1) consumiendo las colonias trasplantadas con un efecto negativo para su supervivencia y (2) reduciendo la competencia con las macroalgas al controlar su crecimento, con un efecto positivo para los trasplantes.

\section{AGRADECIMIENTOS}

Este trabajo es parte del proyecto "Reproducción, crecimiento y supervivencia al trasplante de las especies Montastraea annularis (morfotipo 1), Diploria labyrinthiformis y Porites astreoides (morfo verde) en el Parque Nacional Natural Corales del Rosario, Caribe colombiano" financiado a E. Alvarado, J.A. Sánchez y R. García, por la Universidad Jorge Tadeo Lozano y COLCIENCIAS (120209-221-96). Agradecemos especialmente a C. Gómez (UAESPNN, Minambiente), J.A. Sánchez, R. García, M.F. Gil, O.L. Arenas, H. Charry, C. Sareztki, M. Morelos, Ecobuzos (Cartagena) y Sport Barú (Ciénaga de Cholón) su colaboración en el trabajo de campo. A.G. Morales, S. Correa y S. Lozano por revisar el manuscrito original. Contribución número 59 del Centro de Investigaciones Científicas de la Universidad de Bogotá Jorge Tadeo Lozano.

\section{RESUMEN}

Para investigar el efecto de los peces herbívoros y coralívoros en la supervivencia de colonias trasplantadas de Montastraea annularis, Diploria labyrinthiformis y Porites astreoides se encerraron colonias trasplantadas y nativas en jaulas y se compararon con colonias libres (control), mientras los efectos del encierro fueron evaluados con un tratamiento de techos. Para saber si el estrés debido al trasplante aumentó la vulnerabilidad de los corales a la depredación por peces, se comparó la intensidad de forrajeo por peces entre colonias trasplantadas y nativas. Se realizaron censos visuales para determinar las densidades de las poblaciones de peces herbívoros y coralívoros en la zona de trasplante. Los peces herbívoros móviles (Scaridae y Acanthuridae) fueron los más abundantes, los peces coralívoros (principalmente Chaetodontidae) fueron los más escasos. Se observó un efecto negativo de los peces herbívoros territoriales en la supervivencia de los trasplantes de M. annularis, principalmente al inicio del estudio. La presión de forrajeo fue similar en colonias trasplantadas y nativas, pero fue diferente entre especies, siendo $D$. labyrinthiformis la menos depredada. En los tratamientos de colonias protegidas en jaulas se observó un crecimiento acelerado de macroalgas debido a la reducción del forrajeo por peces herbívoros. Esto causó blanqueamiento parcial y mortalidad parcial en algunas colonias, principalmente de $P$. astreoides. En M. annularis y D. labyrinthiformis no se observaron diferencias significativas en los porcentajes de tejido sano de las colonias entre los tratamientos de jaulas y de control, pero sí en $P$. astreoides. Los resultados sugieren un efecto negativo de los peces herbívoros territoriales en la supervivencia de las colonias durante los primeros días del trasplante; este efecto puede variar dependiendo de las especies de peces y corales involucradas. Los resultados también indican un efecto positivo del forrajeo por peces, debido a la reducción de la presión competitiva alga-coral, sobretodo en $P$. astreoides. Los scáridos parecen afectar la supervivencia de los corales tanto de manera negativa, al morder en el tejido vivo, como de manera positiva al controlar el crecimiento de las macroalgas. En general, el éxito del transplante de coral fue poco afectado por la actividad de forrajeo de los peces, aunque algunas diferencias entre las especies de coral parecen estar relacionadas con la forma de las colonias. La interacción entre el forrajeo de peces hervíboros y el balance competitivo alga-coral puede ser importante en determinar la supervivencia al transplante. 
Key words: peces herbívoros, peces coralívoros, Montastraea annularis, Diploria labyrinthiformis, Porites astreoides, interacción coral-pez.

\section{REFERENCIAS}

Alvarado, E.M. \& M.C. Corchuelo. 1992. Los nutrientes, la temperatura y la salinidad provenientes del Canal del Dique como factores de deterioro en el Parque Nacional Natural Corales del Rosario (Cartagena, Colombia), p. 277-287. In Memorias del VIII Seminario Nacional de Ciencias y Tecnologías del Mar. Comisión Colombiana de Oceanografía, Bogotá, Colombia.

Alvarado, E.M., F. Duque, L. Flórez \& R. Ramírez. 1986. Evaluación cuantitativa de los arrecifes coralinos de las Islas del Rosario (Cartagena-Colombia). Bol. Ecotrópica 15: 1-30.

Bouchon, C., J. Jaubert \& Y. Bouchon-Navaro. 1981. Evolution of a semi-artificial reef built by trasplanting coral heads. Tethys 10: 173-176.

Brock, R.E. 1979. An experimental study on the effects of grazing by parrotfishes and role of refuges in benthic community structure. Mar. Biol. 51: 381-388.

Brown, B.E. 1997a. Disturbances to reefs in recent times, p. 354-379. In C. Birkeland (ed.). Life and death of coral reefs. Chapman \& Hall, Nueva York, EEUU.

Brown, B.E. 1997b. Coral bleaching: causes and consequences. Coral Reefs 16: S129-S138.

Bruckner, A.W. \& R.J. Bruckner. 1998. Destruction of coral by Sparisoma viride. Coral Reefs 17: 350 .

Bruggemann, J.H., M.J.H. van Oppen \& A.M. Breeman. 1994. Foraging by the stoplight parrotfish Sparisoma viride. I. Food selection in different, socially determined habitats. Mar. Ecol. Progr. Ser. 106: 41-55.

Carpenter, R.C. 1986. Partitioning herbivory and its effects on coral reef algal communities. Ecol. Mon. 56: 345-363.

Carpenter, R.C. 1990. Mass mortality of Diadema antillarum. II. Effects on population densities and grazing intensity of parrotfishes and surgeonfishes. Mar. Biol. 104: $79-86$

Clark, S. \& A.J. Edwards. 1995. Coral transplantation as an aid to reef rehabilitation: evaluation of a case study in the Maldive Islands. Coral Reefs 14: 201-213.
Cox, E.F. 1986. The effects of a selective corallivore on growth rates and competition for space between two species of Hawaiian corals. J. Exp. Mar. Biol. Ecol. 101: 161-174.

Fowler, A.J. 1987. The development of sampling strategies for population studies of coral reef fishes. A case study. Coral Reefs 6: 49-58.

Frydl, P. 1979. The effect of parrotfish (Scaridae) on coral in Barbados. W. I. Int. Rev. Ges. Hydrobiol. 64: 737-748.

García, R. 1994. Crecimiento, supervivencia, regeneración y reproducción de la especie Acropora palmata en el Parque Nacional Natural Corales del Rosario. Tesis de pregrado, Universidad Jorge Tadeo Lozano, Cartagena, Colombia. 126 p.

Glynn, P.W. 1990. Feeding ecology of selected coral-reef macroconsumers: patterns and effects on coral community structure, p. 365-399. In Z. Dubinsky (ed.). Ecosystems of the World 25: Coral Reefs. Elsevier, Amsterdam, Holanda.

Guzmán, H.M. 1988. Distribución y abundancia de organismos coralívoros en los arrecifes coralinos de la Isla del Caño, Costa Rica. Rev. Biol. Trop. 36: 191-207.

Guzmán, H.M. 1991. Restoration of coral reefs in Pacific Costa Rica. Cons. Biol. 5: 189-195.

Hay, M.E. \& P.R. Taylor. 1985. Competition between herbivorous fishes and urchins on Caribbean reefs. Oecologia 65: 591-598.

Hughes, T.P. 1994. Catastrophes, phase shifts, and largescale degradation of a Caribbean coral reef. Science 265: $1547-1551$

Kaufman, L.S. 1977. The three-spot damselfish: effects on benthic biota of Caribbean coral reefs, p. 559-564. In Proc. Third Int. Coral Reef Symp. ISRS, Miami, EEUU.

Knowlton, N., J.C. Lang \& B.D. Keller. 1990. Case study of natural population collapse: post-hurricane predation on Jamaican staghorn corals. Smithsonian Contributions Mar. Sci. 31. SI 1. 41: 31.

Lapointe, B.E. 1989. Caribbean coral reefs: Are they becoming algal reefs? Sea Frontiers: 83-91.

Lewis, S.M. 1986. The role of herbivorous fishes in the organization of a Caribbean reef community. Ecol. Mon. 56: 183-200. 
Littler, M.M., P.R. Taylor \& D.S. Littler. 1989. Complex interactions in the control of coral zonation on a Caribbean reef flat. Oecologia 113: 231-238.

McCook, L.J. 1999. Macroalgae, nutrients and phase shifts on coral reefs: scientific issues and management consequences for the Great Barrier Reef. Coral Reefs 18: 357-367.

Miller, M.W. \& M.E. Hay. 1998. Effects of fish predation and seaweed competition on the survival and growth of corals. Oecologia 113: 231-238.

Neudecker, S. 1979. Effects of grazing and browsing fishes on the zonation of corals in Guam. Ecology 60: 666-672.

Ogden, J.C. \& P.S. Lobel. 1978. The role of herbivorous fishes and urchins in coral reef communities. Environ. Biol. Fish. 3: 49-63.

Potts, D.C. 1977. Suppression of coral populations by filamentous algae within damselfish territories. J. Exp. Mar. Biol. Ecol. 28: 207-216.

Ramírez, A. 1986. Ecología descriptiva de las llanuras madreporarias del Parque Nacional Submarino Los Corales del Rosario (Mar Caribe) Colombia. Bol. Ecotrópica 14: 34-63.
Sarmiento, D.E., F.A. Flechas \& G.A. Alvis. 1990. Evaluación cuantitativa del estado actual de las especies coralinas del Parque Nacional Natural Corales del Rosario, Cartagena (Colombia), p. 303-315. In Memorias del VII Seminario Nacional de Ciencias y Tecnologías del Mar. Comisión Colombiana de Oceanografía, Cali, Colombia.

Schonwald, N. 1998. Distribución y composición de los hábitat marinos asociados a las estructuras arrecifales del área de Isla Grande, Archipiélago del Rosario, Caribe colombiano. Tesis de pregrado, Universidad de los Andes, Bogotá, Colombia. $57 \mathrm{p}$.

van Treeck, P. \& H. Schuhmacher. 1997. Initial survival of coral nubbins transplanted by a new coral transplantation technology-options for reef rehabilitation. Mar. Ecol. Prog. Ser. 150: 287-292.

Wellington, G.M. 1982. Depth zonation of corals in the gulf of Panama: control and facilitation by resident reef fishes. Ecol. Mon. 52: 223-241.

Wilkinson, C. 2000. Status of Coral Reefs of the World: 2000. Aust. Int. Mar. Sci. 363 p.

Zar, J.H. 1996. Biostatistical analysis. Prentice-Hall, Nueva Jersey, EEUU. 662 p. 
